\title{
Labia Majora Share
}

\author{
Hanjing Lee ${ }^{1}$, Yan Lin Yap ${ }^{1}$, Jeffrey Jen Hui Low ${ }^{2}$, Jane Lim ${ }^{1}$ \\ ${ }^{I}$ Division of Plastic, Reconstructive and Aesthetic Surgery, Department of General Surgery, ${ }^{2}$ Department of Obstetrics and Gynaecology, \\ National University Health System, Singapore
}

Defects involving specialised areas with characteristic anatomical features, such as the nipple, upper eyelid, and lip, benefit greatly from the use of sharing procedures. The vulva, a complex 3-dimensional structure, can also be reconstructed through a sharing procedure drawing upon the contralateral vulva. In this report, we present the interesting case of a patient with chronic, massive, localised lymphedema of her left labia majora that was resected in 2011. Five years later, she presented with squamous cell carcinoma over the left vulva region, which is rarely associated with chronic lymphedema. To the best of our knowledge, our management of the radical vulvectomy defect with a labia majora sharing procedure is novel and has not been previously described. The labia major flap presented in this report is a shared flap; that is, a transposition flap based on the dorsal clitoral artery, which has consistent vascular anatomy, making this flap durable and reliable. This procedure epitomises the principle of replacing like with like, does not interfere with leg movement or patient positioning, has minimal donor site morbidity, and preserves other locoregional flap options for future reconstruction. One limitation is the need for a lax contralateral vulva. This labia majora sharing procedure is a viable option in carefully selected patients.

Keywords Perineum / Lymphedema / Carcinoma, squamous cell
Correspondence: Jane Lim

Division of Plastic Reconstructive and Aesthetic Surgery, Department of General Surgery, National University Health System, Singapore Tel: $+65-67725555$

Fax: $+65-67722336$

E-mail: cfslimj@nus.edu.sg

Received: 14 Aug $2016 \bullet$ Revised: 21 Sep $2016 \bullet$ Accepted: 27 Sep 2016

pISSN: 2234-6163 • elSSN: 2234-6171 • https://doi.org/10.5999/aps.2017.44.1.80 • Arch Plast Surg 2017;44:80-84

\section{INTRODUCTION}

The beauty of the specialty of plastic specialty lies in creativity and innovation. The reconstruction of defects created during resection is based on a solid grounding of surgical principles and a detailed anatomical understanding of anatomy. Replacing like with like is an important guideline when designing and planning our reconstructions, and this is epitomized in the sharing. Specialised areas with characteristic anatomical features benefit greatly from sharing procedures, as it is otherwise difficult to reproduce the features and structures seen in the eyelids, lips, and nipples. Sharing and cross flaps are similar in that both can be forms of transposition flaps. However, in cross flaps, division of the pedicle is required at a later stage. Sharing has the additional benefit of not requiring a second operation for division.

The vulva is a complex urogenital region of the perineum that comprises the mons pubis, labia majora, labia minora, clitoris, urinary meatus, vaginal introitus, and vestibule. Reconstruction of this 3-dimensional region is challenging because the external structure is intimately related to key features of urinary and bowel continence, reproduction, and sexual function. As such, maintenance of sensation is also a crucial consideration. The ability to restore an aesthetically acceptable appearance has a pronounced impact on patients' self-worth, confidence, and psychosexual outcomes.

The necessity of reconstructing the vulva usually arises from oncologic ablation, congenital anomalies, or traumatic injury, which includes postsurgical deformities, as exemplified by la- 
bioplasty complications [1,2]. Commonly practiced approaches include the pudendal-thigh flap (Singapore flap) and its modifications, the internal pudendal artery perforator flap, the gracilis myocutaneous flap, the inferior gluteal flap, the vertical rectus abdominis musculocutaneous flap, and the anterolateral thigh flap. Each of these commonly utilized methods is associated with specific complications, such as obliteration of the groin fold when the flap is continuous with the thigh and the inability to reproduce labia with comparable symmetry, shape, and consistency to the contralateral side.

Massive localized lymphedema is a long-standing and slowgrowing soft-tissue pseudotumour characterized by prominent oedema, fibrillary collagen, and uniformly distributed fibroblast and vascular proliferation in the adipose tissue. This condition was first described in 1998, predominantly in the limbs of 14 patients with morbid obesity [3]. Squamous cell carcinoma (SCC) arising from chronic lymphedema is a rare entity; since its first description in 1981, only 16 cases have been reported in the literature, with none involving the labia [4].

Here, we present the first reported case of left labia chronic lymphedema with SCC involvement, as well as our unique reconstruction using a labia majora share.

\section{IDEA}

A 50-year-old patient first presented to us in 2011 with massive localized lymphedema of her left labia majora. The lymphedema first appeared in her early 30s and rapidly increased in size from 2008 to 2011, affecting her mobility (Fig. 1). The resected left labia lymphedema specimen weighed $915 \mathrm{~g}$, and the histo-

\section{Fig. 1. Massive localized chronic lymphoedema}

Appearance of the left labia majora massive localized chronic lymphoedema in 2011, prior to surgical resection. The resection specimen weighed $915 \mathrm{~g}$.

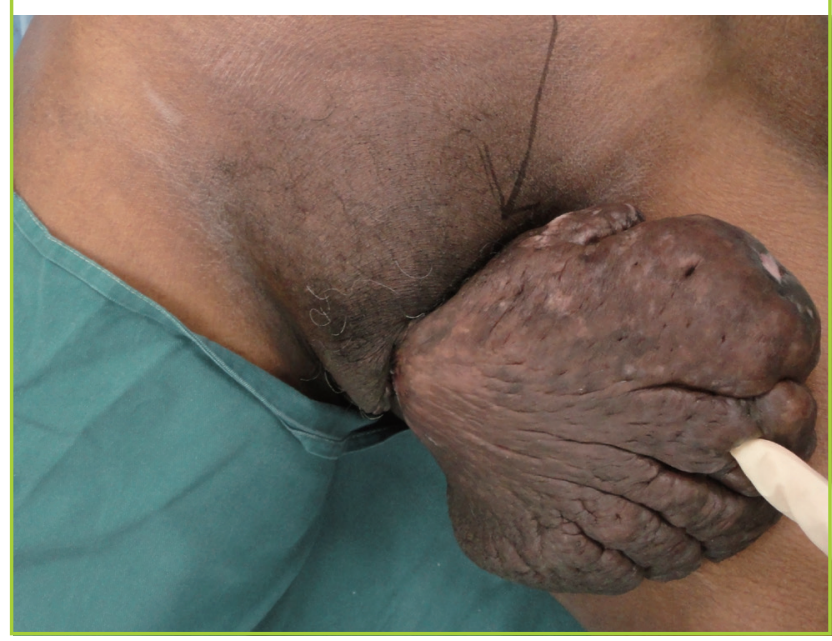

logical report showed chronic lymphedema with no evidence of malignancy. A case of mild concomitant right labia minora swelling was resected at the same time as a balancing procedure. The patient's other pertinent medical history included ulcerative colitis and metastatic left breast cancer, for which bilateral mastectomy, left axillary clearance, and a right sentinel lymph node biopsy were performed in 2011. She defaulted surgical follow-up until 2014, when an ulcer was noted in vicinity of her left labia by her oncologist. She refused further investigation of the ulcer for the following 2 years until the size, pruritis, pain, discharge, and contact bleeding became debilitating. A $6 \times 8-\mathrm{cm}$ fungating and lichenified lesion over her left vulva, abutting the clitoris and the vaginal wall, was apparent on clinical examination without clinically palpable inguinal lymph nodes. Her right vulva was noted to be significantly bulky despite the previous resection (Fig. 2). Computed tomography scans showed uterine fibroids, a positron emission tomography scan highlighted the left vulva lesion as well as the deep left inguinal lymph nodes, and a bone scan did not reveal any bony destruction. The biopsy samples were determined to be SCC.

The patient was placed in the lithotomy position with her legs in stirrups. The bladder was catheterized. Following radical vulvectomy with 2 -cm radial margins and bilateral inguino-femoral lymphadenectomy, a $12 \times 8 \mathrm{~cm}$ defect was created, involving the whole of the left labia majora, the clitoris, and extending medially to the vestibule. The mons pubis was spared.

The right side of the labia was used for sharing in a superiorly based transposition flap based on the right dorsal clitoral artery. The overlying skin was mildly thickened, but no signs of hyperkeratosis or lymphangiectasis were present, and the underlying

\section{Fig. 2. Left vulvar squamous cell carcinoma}

Left-sided vulvar fungating squamous cell carcinoma abutting the clitoris and the vaginal wall. The right side of the vulva is notably bulky, and the scar from the previous labia minora resection is visible.

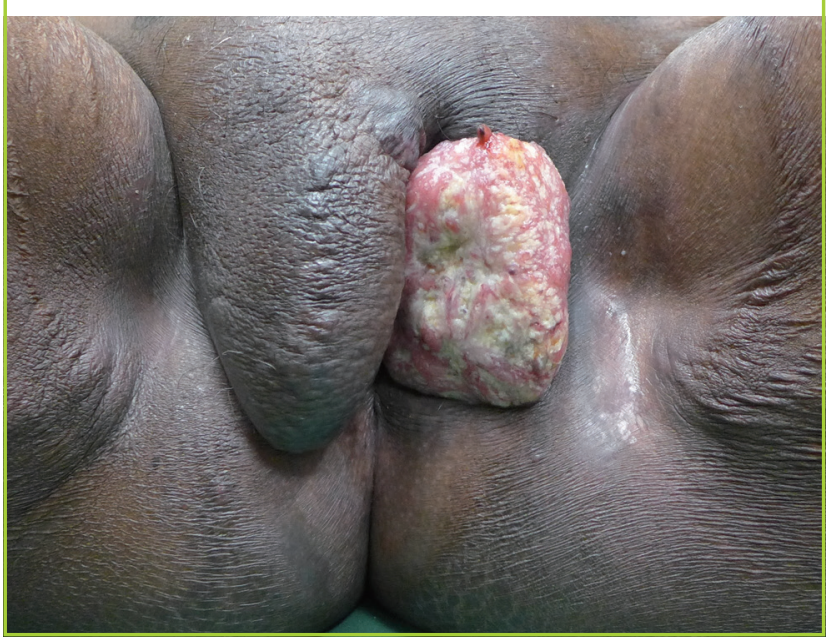




\section{Fig. 3. Labia majora sharing procedure}

(A) Intraoperative indocyanine green fluorescence imaging showing good perfusion of the whole flap prior to sharing. (B) Labia majora flap transposed to cover the defect with primary closure of the donor site. (C) Immediate postoperative picture.
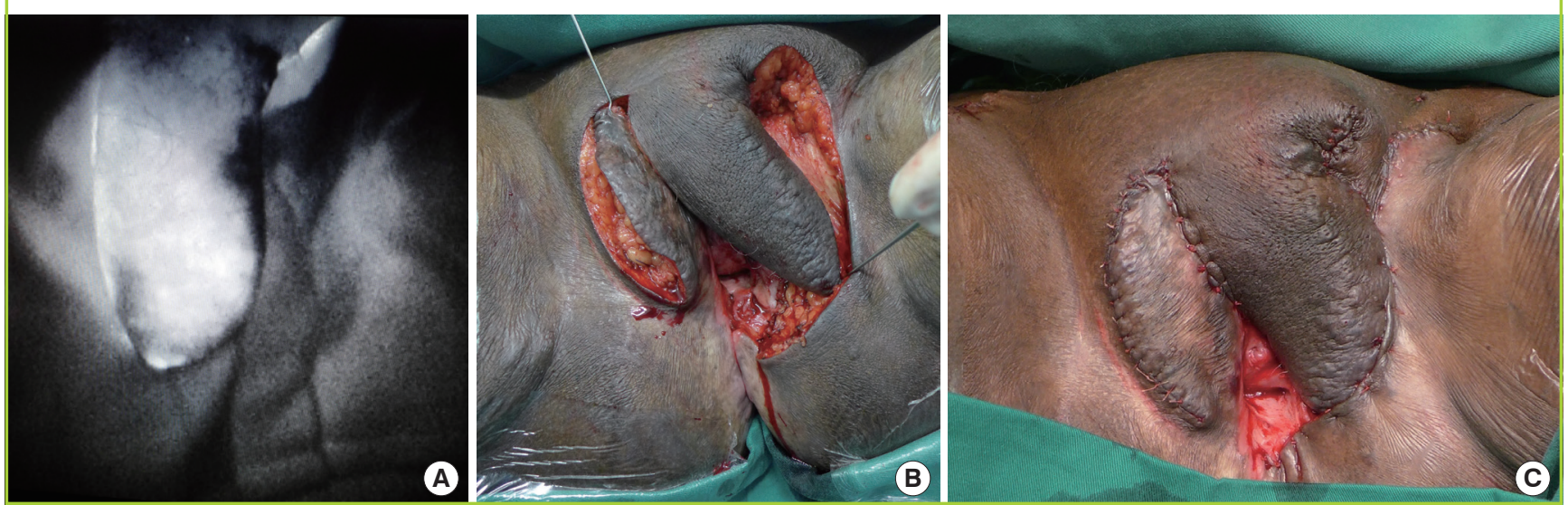

Fig. 4. Labia majora sharing procedure, postoperative

$(A, B)$ Two months following the operation, the symmetry and bulk of both sides of the labia majora were satisfactory in both supine and standing positions. No deviations in the patient's urinary flow were noted.
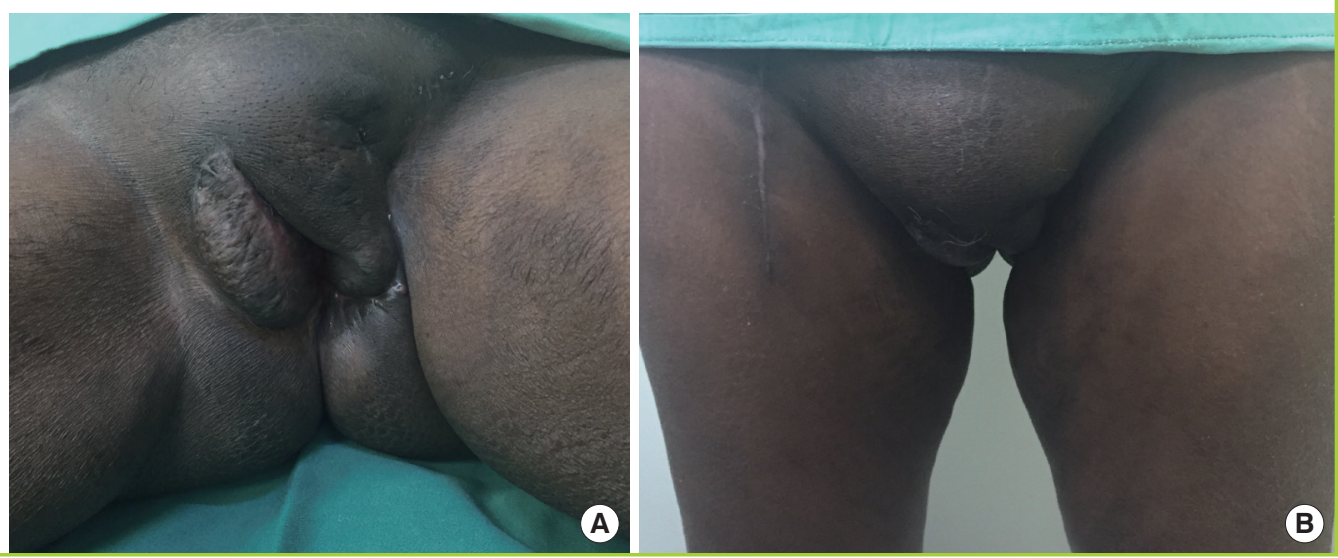

subcutaneous tissue was normal, without fibrosis or edema. Preoperatively, the right branch of the dorsal clitoral artery supplying the right labia was marked out using a hand-held Doppler. The incision began at the tip of the flap posteriorly and deepened through the skin and subcutaneous tissue down to the loose areolar tissue on both sides of the labia majora. The flap elevation proceeded anteriorly towards the mons pubis and its neurovascular supply. The dorsal clitoral artery was identified within its subcutaneous pedicle and preserved. Over the midline, the suspensory ligament of the clitoris was transected to facilitate flap transposition. The flap was transposed $70^{\circ}$ to the left vulva defect. Indocyanine green fluorescence imaging was performed prior to and after flap inset, showing excellent vascularity of the flap. The donor site was closed primarily (Fig. 3). The tumour was found to be a stage 3, well-to-moderately-differentiated SCC with clear resection margins. Herpes simplex virus 16 and 18 were not detected on genotyping. The wound healed without complications and no deviation was observed in her micturition stream (Fig. 4).

\section{DISCUSSION}

The anatomy of the vulva region varies widely, and was nicely depicted by the contemporary artist Jamie McCartney in his The Great Wall of Vagina, in which plaster casts of 400 women's vulvas are on display. Clinically, imaginary lines are drawn between the pubic symphysis and the 2 ischial tuberosities, forming the urogenital triangle. It contains the mons pubis fat pad, which extends posteriorly, forming the 2 labia majora that merge once again in the perineum. Medially to the labia majora are the hairless labia minora, which fuse anteriorly, forming the prepuce and frenulum of the clitoris. The vestibule then extends medially to the hymenal sulcus. The external urethral meatus and the introitus of the vagina and accessory glands reside inside these structures.

The arterial supply of this region originates from both the ex- 


\section{Fig. 5. Vascular supply of the labia majora flap}

The dorsal clitoral artery, one of the distal branches of the internal pudendal artery, has perforating branches that supply the subcutaneous labia majora flap.

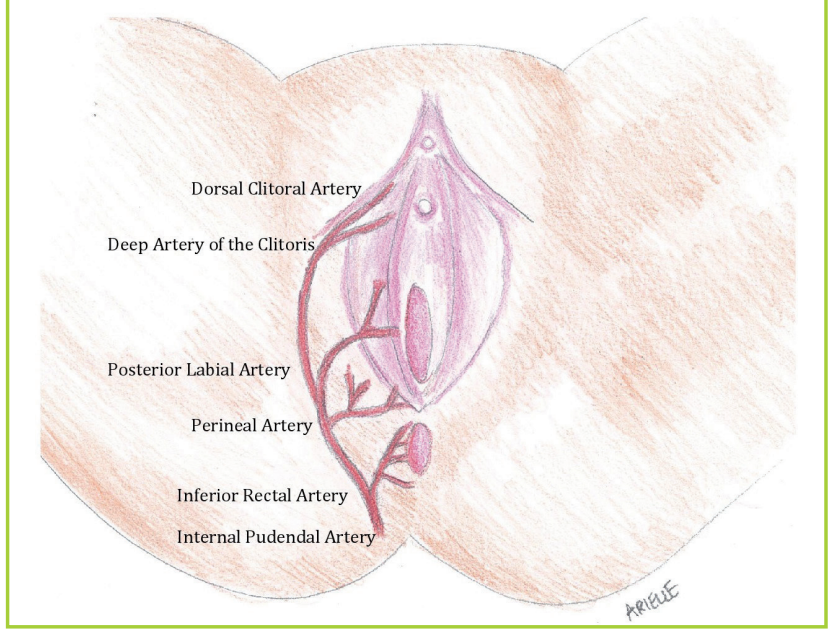

ternal and internal pudendal arteries. The external pudendal artery, which arises from the femoral artery, continues within the labia as the anterior labial artery. From the internal pudendal artery, the posterior labial artery supplies the posterior portion of the labia and the dorsal artery of the clitoris, while the deep artery of the clitoris supplies the anterior portion. This artery runs deep to the deep fascia on the dorsum of the clitoris, and therefore the elevation of our subcutaneous labia majora flap was based on the perforating branch of the dorsal artery of the clitoris, which was located using hand-held Doppler, with good vascularity shown in indocyanine green fluorescence imaging (Fig. 5 ). The venous drainage is from the labial veins, which are tributaries of the internal pudendal veins, as well as the venae comitantes of the internal pudendal artery.

Chronic lymphedema has been reported to be a carcinogenic factor, contributing to the development of malignancy [5]. Vascular tumours, such as Stewart-Treves lymphangiosarcoma and Kaposi sarcoma, are more commonly associated with chronic lymphoedema due to the continual angiogenic stimulus [6]. However, a variety of other tumours have also been reported in the literature in association with chronic lymphoedema, including basal cell carcinoma, malignant melanoma, lymphoma, and epidermoid carcinoma. SCC has rarely been found to be associated with chronic lymphedema. So far, 4 congenital cases and 9 acquired cases, all arising in the extremities $[7,8]$, as well as 3 cases with penoscrotal involvement [9-11], have been reported. To the best of our knowledge, this study is the first to report involvement of the labia. In contrast, in the absence of chronic lymphedema, $95 \%$ of all vulva cancers are SCC $[12,13]$. The scarcity of the published literature on SCC in association with chronic lymphedema could possibly reflect under-diagnosis or under-reporting of this condition. This diagnosis should be considered in patients presenting with ulceration over a chronic lymphedema site.

Localized T1 vulvar cancer can be adequately managed with wide local excision. Radical vulvectomy (hemivulvectomy, anterior or posterior vulvectomy) is performed for $\mathrm{T} 2$ or larger lesions with involvement of the urethra, vaginal wall, and/or anus, and reconstruction of the resultant defect is necessary. Multiple authors have proposed reconstructive algorithms for partial or complete vulvar defects $[14,15]$. Vulva and labial defects are usually cutaneous or subcutaneous in nature. The goal of reconstruction is to create 2 adequately sized and sensate labial folds that do not negatively affect the patient's psychosexual image or interfere with the functions of micturition or sexual intercourse. Reliable soft tissue reconstruction should also ensure short hospitalization and quick rehabilitation.

Small defects can be closed primarily or with local tissue repositioning. Reconstruction with a split-thickness or full-thickness skin graft is another simple option for superficial vulvectomy, or if the risk of local recurrence is high, provided that no prior irradiation has been performed and that postoperative radiation therapy is not planned, due to the high rate of skin graft loss. Another limitation to the use of those grafts is that they tend to contract and distort the urethra or the introitus, with resultant functional and aesthetic sequelae. Larger defects require local flap coverage, which could be accomplished using either random-pattern flaps or axially based fasciocutaneous flaps. The pudendal-thigh flap (Singapore flap), which is based on the posterior labial artery and its anastomosis to the branches of the deep external pudendal artery, medial femoral circumflex artery, and anterior branch of the obturator artery, is frequently performed due to its simplicity of execution and reliability. The more posteriorly located internal pudendal artery perforator flaps, designed as propeller, transposition, or V-Y advancement flaps, have been shown to be options for reliably reconstructing the vulva, vagina, and buttocks. Should local flap options be nonviable as a result of tumour involvement or previous irradiation, regional fasciocutaneous or musculocutaneous flaps are alternatives. Medial thigh flaps based on the superficial femoral artery and anterolateral thigh flaps have been used to provide pliable tissue for resurfacing. Musculocutaneous flaps such as the gracilis flap, vertical rectus abdominis flap, gluteal thigh and tensor fascia lata flap, are generally bulkier and generally require debulking for resurfacing of the vulvar region. Furthermore, flap viability may be compromised due to mobilization of the legs and stretching of the pedicle.

The benefits of the labia majora share are that it has a consis- 
tent vascular anatomy based on the dorsal clitoral artery, which ensures its reliability and durability. The dorsal clitoral artery supplies the distal tip of the flap, which covered the posteriormost portion of the labia majora, as shown in the indocyanine green images. The length of the flap is sufficient for coverage up to the posterior border of the urogenital triangle, without having to dissect the perforator artery, making this a technically simple and fast flap harvest. It also allows for symmetricalisation, if the contralateral labia majora is of sustainable bulk. By replacing like with like, it retains the characteristic soft pliable nature and hairbearing quality of this tissue, as well as a similar pigmented hue. Since it is based in the urogenital region, wound management and cleaning are facilitated, as no additional distal donor site needs to be managed. Neither leg movement nor patient position has any impact on wound tension or subsequent wound healing. Primary closure of the donor site minimizes donor site morbidity. Finally, it preserves other local and regional flap options for future reconstruction should the need arise. The only disadvantage precluding its use is the potential lack of bulk in the labia majora in some patients.

In conclusion, SCC developing on the background of massive localized chronic lymphedema is a rare entity. A chronic wound with malignant features should always raise the suspicion of SCC. The labia majora share is shown to be an elegant option for functional vulva reconstruction, providing the best match in terms of tissue consistency, pigmented skin colour, hair-bearing characteristics, and contour, with minimal donor site involvement. This technique does not require a second operation for pedicle division. The limitations of its use are the need for sizable bulk or laxity in the contralateral labia, which are more common in slightly older populations. The sensibility of this shared labia flap has yet to be determined.

\section{REFERENCES}

1. Nguyen AT, Ramsden AJ, Corrigan BE, et al. Labial reconstruction with a cross-labial flap. J Plast Reconstr Aesthet Surg 2011;64:1383-5.

2. Zeplin PH, Nuwayhid R, Nuwayhid M. Two-stage posterior cross-labial transposition flap: a novel technique for labium minus reconstruction. Aesthetic Plast Surg 2014;38:930-2.
3. Farshid G, Weiss SW. Massive localized lymphedema in the morbidly obese: a histologically distinct reactive lesion simulating liposarcoma. Am J Surg Pathol 1998;22:1277-83.

4. Shelley WB, Wood MG. Transformation of the common wart into squamous cell carcinoma in a patient with primary lymphedema. Cancer 1981;48:820-4.

5. Furukawa H, Yamamoto Y, Minakawa H, et al. Squamous cell carcinoma in chronic lymphedema: case report and review of the literature. Dermatol Surg 2002;28:951-3.

6. Ruocco V, Schwartz RA, Ruocco E. Lymphedema: an immunologically vulnerable site for development of neoplasms. J Am Acad Dermatol 2002;47:124-7.

7. Gomes CA, Magalhaes CB, Soares C Jr, et al. Squamous cell carcinoma arising from chronic lymphedema: case report and review of the literature. Sao Paulo Med J 2010;128:42-4.

8. Parthiban R, Kaler AK, Shariff S, et al. Squamous cell carcinoma arising from congenital lymphedema. SAGE Open Med Case Rep 2013;1:2050313X13496507.

9. Hadway P, Lynch M, Corbishley CM, et al. Squamous cell carcinoma of the penis in a patient with chronic isolated penile lymphoedema. Urol Int 2006;76:87-8.

10. Abhyankar SV, Kulkarni A, Kulkarni M, et al. Squamous cell carcinoma of the penis and scrotum in a patient with chronic scrotal and penile lymphedema. Indian J Dermatol 2010; 55:387-9.

11. Su TF, Lee HS, Gao HW, et al. Squamous cell carcinoma arising from massive localized lymphedema of scrotum mimicking scrotal smooth muscle hamartoma of dartos: a case report. Am J Dermatopathol 2015;37:551-4.

12. Sideri M, Jones RW, Wilkinson EJ, et al. Squamous vulvar intraepithelial neoplasia: 2004 modified terminology, ISSVD Vulvar Oncology Subcommittee. J Reprod Med 2005;50: 807-10.

13. Alkatout I, Schubert M, Garbrecht N, et al. Vulvar cancer: epidemiology, clinical presentation, and management options. Int J Womens Health 2015;7:305-13.

14. Hockel M, Dornhofer N. Vulvovaginal reconstruction for neoplastic disease. Lancet Oncol 2008;9:559-68.

15. Hage JJ, Beurden M. Reconstruction of acquired perineovulvar defects: a proposal of sequence. Semin Plast Surg 2011;25:148-54. 